\title{
Body image, weight discrepancy and body mass index among university students in Riyadh, Saudi Arabia
}

\author{
OLUMATOYIN O. TORIOLA ${ }^{1}$ AND SUNDAY O. ONAGBIYE ${ }^{2}$
}

IPrince Sultan University, Women Campus, Health and Physical Education Department, Riyadh,
Kingdom of Saudi Arabia
'Department of Sport, Recreation and Exercise Science, University of the Western Cape,
Bellville, South Africa

(Received: 18 August 2020; Revision Accepted:24 October 2020)

ORCID nos.: OOT (0000-0003-4677-0433), SOO (0000-0003-4931-007X)

DOI: https://doi.org/10.37597/ajphes.2020.26.4.7

\begin{abstract}
This study examined the relationship between body image, weight discrepancy and body mass index among female university students in Riyadh, Saudi Arabia. A total of 183 university students aged $18.7 \pm 1.17$ years volunteered to participate in the study. Anthropometric variables were measured according to the protocol of International Society for the Advancement of Kinanthropometry (ISAK). Body image perception was assessed using silhouette matching techniques. Multiple regression analyses were performed to examine the relationships between body image and weight perceptions in relation to actual physical measurements by means of two indices: Feel minus Ideal Discrepancy (FID) and FAI (Feel weight status minus actual weight status inconsistency). One-way ANOVA was computed to test for any substantial differences in the participants' dependent measures according to body mass index (BMI) categories. Percentage distribution of participants' actual weight categories were $71 \%$ (underweight), 18.6\% (normal weight) and $10.4 \%$ (overweight). Using the body silhouette chart, the mean value for the participants' feel and ideal figures were 3.6 and 2.2, while their FID and FAI scores were 1.4 and 0.55 , respectively. Results of the regression analysis indicated that every unit increase in Feel figure yielded a highly significant increase in BMI by $1.4 \mathrm{~kg} / \mathrm{m}^{2}$. By contrast, a unit increase in the Ideal figure resulted in a non-significant decrease in BMI by $-0.19 \mathrm{~kg} / \mathrm{m}^{2}$. The relationship between the actual body weight and body image discrepancy among Riyadh university students has practical implications for their health.
\end{abstract}

Keywords: Body image perception, body mass index, women, University, Saudi Arabia.

\section{How to cite this article:}

Toriola, O.O. \& Onagbiye, S.O. (2020). Body image, weight discrepancy and body mass index among university students in Riyadh, Saudi Arabia. African Journal for Physical Activity and Health Sciences, 26(4), 438-447. DOI: https://doi.org/10.37597/ajphes.2020.26.4.7

\section{Introduction}

Body image and weight perception have been understood to be based on selfopinion which could have its roots in both psychological and cultural factors (Mintem, Horta, Domingues \& Gigante, 2015). The influences of appealing body image advertisement on mass media have resulted in many youths being 
pressurised about their body image, and how they perceived themselves (Zaccagni, Masotti, Donati, Mazzoni \& Gualdi-Russo, 2014). Body image concept could be divided into three aspects, which involve the understanding of how a person thinks, feels, and wants to look (As-Sa'edi, Sheerah, Al-Ayoubi, Tajaddin \& Habeeb, 2013). Individual perception of their weight status and body image is often influenced by one's imagination of their own body (As-Sa'edi et al., 2013). Body size perception can be defined as the image that people form in their mind of how they appear physically, which then symbolises the extent of their satisfaction based on their weightiness and figure (Albeeybe, Alomer, Alahmari, Asiri, Alajaji, Almassoud \& Al-Hazzaa, 2018).

Previous studies have reported that persons who are dissatisfied with their body image have a higher likelihood of engaging in positive conduct that could promote body weight control (Zaccagni et al., 2014; Radwan, Hasan, Ismat, Hakim, Khalid, Al-Fityani, Mohammed \& Ayman, 2019). However, unwanted body image is also associated with psycho-physiological sufferings and could adversely influence the quality of life for many (Bouzas, Bibiloni \& Tur, 2019).

A relationship exists between body image and body weight among individuals. In an Indian study conducted among female college students, a significant relationship was reported between the students' body image and shape status, which was directly associated with their lifestyle (Srinivas, Ravi, Prashantha \& Prakash, 2017). Srinivas et al. (2017) further indicated that an increase in body mass index (BMI) (a composite index used to evaluate obesity calculated by dividing weight by height squared: weight $[\mathrm{kg}] /$ height $[\mathrm{m}]^{2}$ ) could trigger the efforts towards preferred bodily form. The imprecise view of one's body weight and weight perception was connected to how body weight is managed (Alwan, Viswanathan, Paccaud \& Bovet, 2011). Factors such as age, gender, values, education levels, socio-economic status, and BMI have all been reported to be associated with bodyweight perception. Radwan et al. (2019) were of the opinion that women compared to men are more likely to wrongly distinguish their weightiness.

Studies on body weight satisfaction are usually based on Higgins' (1987) SelfDiscrepancy Theory (SDT) (Strauman, Vookles, Berenstein, Chaiken \& Higgins (1991). The SDT posits that problems associated with body dissatisfaction and disordered eating could affect self-concept. According to SDT, people experience different types of emotional discomfort arising from incompatible self-concepts and beliefs. The theory postulates domains of self, including the actual self (i.e. a representation of the attributes that the individual or a significant other believes she possesses), the ideal self (i.e. a depiction of the attributes that the individual or a significant other would ideally like her to possess), and the ought self (which is a representation of the attributes that the individual or a significant other believes it is her obligation or duty to possess) (Higgins, 1987). Therefore, discrepancies 
between the actual self and self-guides or ideal self could lead to negative emotional-motivational states and affect body image.

In Saudi Arabia, a number of studies on body image and perceptions have been conducted (Alhussaini, Alsuwedan, Alnefaie, Almubrek, Aldaweesh, Anitha \& Qassem, 2018; ALAhmari, Alomar, ALBeeybe, Asiri, ALAjaji, ALMasoud \& AlHazzaa, 2019; Khalaf, Westergren, Berggren, Ekblom \& Al-Hazzaa, 2015). However, the relationships between body image and weight perceptions, and actual body measurements among university women in Saudi Arabia have rarely been reported. Such studies are needed to address psychosocial issues concerning body image and BMI, especially among young women. Therefore, this study examined the relationship between body image, weight perceptions and BMI among university students in Riyadh, Saudi Arabia.

\section{Methodology}

\section{Study design and sample}

Using a cross-sectional survey design, this study evaluated the association between body image, weight discrepancy and BMI among 183 apparently healthy female students (mean age: 18.7 \pm 1.17 years) at Prince Sultan University (PSU) Campus for Women in Riyadh, Saudi Arabia, who volunteered to participate in the study. Specifically, the relationship between the participants' body image, weight perceptions and BMI was analysed using the FAI (Feel weight status minus actual weight status inconsistency) index (Zaccagni et al., 2014). The study was carried out from October 2017 - April 2018.

\section{Ethical considerations}

The study was approved by the higher research management of Prince Sultan University Campus for women, Riyadh Saudi Arabia. Before data collection, the purpose and procedures of the study were explained to the women, who were also informed that they could decline to participate in the study without any repercussion. Data were collected from the participants who gave informed consent. Guidelines of the Helsinki declaration for the use of human participants in research (General Assembly of the World Medical Association, 2014) were observed.

\section{Anthropometric measurements}

Height and weight were assessed according to the protocol of the International Society for the Advancement of Kinanthropometry (ISAK) (Marfell-Jones, Stewart \& De Ridder, 2012). Height was measured to the nearest $0.1 \mathrm{~cm}$ in bare feet with participants standing upright against a stadiometer. A digital weighing scale [(Omron body composition monitor (Model no. BF508)] calibrated regularly to the nearest $0.1 \mathrm{~kg}$ was used to measure body weight with participants lightly dressed. Subsequently, body mass index (BMI) was calculated from the 
participants' height and weight measurements; using the formula [weight $(\mathrm{kg}) /$ height $\left.(\mathrm{m})^{2}\right]$. For the purpose of this study, body mass indices were used to classify the participants into the following weight categories expressed as $\mathrm{kg} / \mathrm{m}^{2}$ : Underweight (less than 18.5), normal (18.5-24.9), overweight (25-29.9) or obese (30 and above) (Cole, Flegal, Nicholls \& Jackson, 2007).

\section{Body image assessment}

Body image perception was assessed using the adaptations of the protocol used in Zaccagni et al.'s (2014) study. In this study, the silhouette matching technique often female figures with increasing body weight from figure 1 to figure 10 was shown to the students. The subjects indicated which figure best represented how they presently looked (feel) and how they ideally wanted to look (ideal). Body dissatisfaction was calculated as Feel minus Ideal Discrepancy (FID) (Zaccagni et al., 2014) with negative scores meaning the desire to be fatter and positive scores meaning the desire to be thinner.

Following the protocol used by Zaccagni and colleagues (2014), FAI (Feel weight status minus actual weight status inconsistency) index was created to assess the inconsistency in weight status perception using the silhouette matching technique as an alternative to confirming the presence or absence of a realistic weight status perception in the women based on their body size measurement (BMI) and the Feel figure.

Specifically, FAI was calculated as Feel weight status minus actual weight status inconsistency in which the orthodox code assigned to the actual weight status of the subject (code: 1 for underweight status, 2 for normal weight status, 3 for overweight status, and 4 for obese status) was deducted from the one corresponding to her Feel figure according to the following correspondence: figures 1 and 2 match Feel status 1; figures 3, 4 and 5 match Feel status 2; figures 6 and 7 match Feel status 3; and figures 8, 9 and 10 matches Feel status 4. In Zaccagni et al.'s (2014) study, nine figures were used, but a tenth figure was included in this current study. Therefore, figures 8,9 , and 10 correspond to match Feel status 4 . Furthermore, since the FAI scores range from -3 to +3 , an individual with a negative FAI value was interpreted as having mean weight status underestimation, while those with positive FAI values have overestimated weight status, and an FAI score of 0 was interpreted as an accurate perception of own weight status.

\section{Statistical analyses}

Data analysis was carried out using the IBM statistical package for the social sciences (SPSS) (Statistics for Windows, version 26.0 Armonk, NY: IBM Corporation). The results were expressed as mean values and standard deviations (SD). One-way analysis of variance (ANOVA) was used to test for any considerable differences in the participants' dependent variables according to BMI 


\section{Toriola and Onagbiye}

categories. Multiple linear regression analyses were performed to determine the relationship between the participants' BMI and body image assessment traits. All statistical tests were two-tailed and $\mathrm{p} \leq 0.05$ was considered statistically significant.

\section{Results}

Table 1 provides summary data on the participants' anthropometric characteristics and body image assessments. On average, the participants fell within a normal BMI $\left(18.8 \pm 3.90 \mathrm{~kg} / \mathrm{m}^{2}\right)$ category.

Table 1: Descriptive characteristics of the sample

\begin{tabular}{lllll}
\hline Traits & M & SD & MIN. & MAX. \\
\hline Age (years) & 18.7 & 1.17 & 17 & 24 \\
Weight (kg) & 60.9 & 12.9 & 40 & 105 \\
Height $(\mathbf{c m})$ & 1.62 & 0.06 & 1.47 & 1.78 \\
BMI $\left(\mathbf{k g} / \mathbf{m}^{2}\right.$ ) & 18.8 & 3.90 & 11.98 & 32.21 \\
Feel figure & 3.60 & 2.50 & 0 & 10 \\
Ideal figure & 2.19 & 1.53 & 0 & 10 \\
\hline FID & 1.40 & 2.32 & -4 & 10 \\
FAI & 0.55 & 0.91 & -1 & 3 \\
\hline
\end{tabular}

Feel minus Ideal Discrepancy (FID), FAI (Feel weight status minus Actual weight status Inconsistency)

Further analysis indicated that $71 \%$ of the participants were underweight and $18.6 \%$ were of normal weight, while $10.4 \%$ were overweight. Using the body silhouette chart, the mean values for the participants' Feel and Ideal figures were 3.6 and 2.2, while their FID and FAI scores averaged 1.4 and 0.55 , respectively.

When the participants' body image variables based on BMI categories (BMI < 18.5 , $\mathrm{BMI} \geq 18.5-<25$ and $\mathrm{BMI} \geq 25$ ) were analysed with one-way ANOVA, the main effect of Feel figure $\left(\mathrm{F}_{(2,182)}=<0.001\right)$, Ideal figure $\left(\mathrm{F}_{(2,182)}=0.002\right)$, FID $\left(\mathrm{F}_{(2,182)}=<0.001\right)$ and FIA $\left(\mathrm{F}_{(2,182)}=<0.001\right)$ on BMI were all significant (Table 2). Therefore, as BMI increased, all the traits (feel figure, ideal figure, FID, FIA) also rose concomitantly.

Table 2: Body image assessment by weight status

\begin{tabular}{llllll}
\hline Traits & $\begin{array}{l}\text { BMI Category } \\
\text { BMI }<\mathbf{1 8 . 5} \\
\text { Mean } \pm \text { SD }\end{array}$ & $\begin{array}{l}\text { BMI } \geq \mathbf{1 8 . 5}-<\mathbf{2 5} \\
\text { Mean } \pm \text { SD }\end{array}$ & $\begin{array}{l}\text { BMI } \geq 25 \\
\text { Mean } \pm \text { SD }\end{array}$ & Total & F-ratio \\
\hline Feel figure & $2.42 \pm 1.46$ & $5.68 \pm 2.13$ & $7.89 \pm 1.15$ & $3.60 \pm 2.49$ & $135.1^{* * *}$ \\
Ideal figure & $1.95 \pm 1.28$ & $2.71 \pm 1.56$ & $2.95 \pm 2.40$ & $2.19 \pm 1.53$ & $6.30^{* *}$ \\
FID & $0.47 \pm 1.34$ & $2.97 \pm 2.15$ & $4.95 \pm 2.93$ & $1.40 \pm 2.31$ & $71.9^{* * *}$ \\
FAI & $0.21 \pm 0.57$ & $1.02 \pm 0.79$ & $1.94 \pm 1.22$ & $0.54 \pm 0.91$ & $59.5^{* * *}$ \\
\hline
\end{tabular}

$* * \mathrm{p}<0.01 ; * * * \mathrm{p}<0.001$ (Differences between BMI categories compared using one-way ANOVA). 
The results of the multiple linear regression analysis performed on all participants' data to predict BMI based on Feel and Ideal figures are provided in Table 3.

Table 3: Multiple linear regression analysis to predict BMI and Feel and Ideal figure

\begin{tabular}{lccccc}
\hline Variables & Coefficients & $\mathbf{t}$ & $\boldsymbol{P}$-value & \multicolumn{2}{c}{ Adjusted OR (95\% CI) } \\
\hline Feel figure & 1.370 & 19.978 & $<0.001$ & 1.23 & 1.50 \\
Ideal figure & -0.186 & -1.660 & 0.09 & -0.41 & 0.04 \\
\hline
\end{tabular}

$R^{2}=0.716 ;$ adjusted $R^{2}=0.712: \mathrm{p} \leq 0.05$

The results showed that every unit increase in Feel figure led to a higher significant increase in BMI by $1.4 \mathrm{~kg} / \mathrm{m}^{2}$. This means that the higher the Feel figure, the greater the women's BMI. Furthermore, every unit increase in the ideal figure yielded a non-significant decrease in BMI by $-0.19 \mathrm{~kg} / \mathrm{m}^{2}$. On the contrary, the higher the Ideal figure perceptions, the lower the participants' BMI. Therefore, the results of the regression analysis indicated that the two predictors combined explained $71.6 \%$ of the variance $\left(R^{2}=0.716, \mathrm{~F}(2,182)=226.402, \mathrm{p}<0.001\right)$ in BMI. However, it was found that while the Feel figure significantly predicted BMI $(b=1.37, \mathbf{p}<0.001)$, the Ideal figure's prediction was non-significant $(b=-0.186$, $\mathrm{p}<0.09$ ).

\section{Discussion}

This study examined the relationship between the actual body measurements, body image and weight discrepancy among university students in Riyadh, Saudi Arabia. Despite the finding which showed that the participants had an overall normal body mass index (IOTF's BMI classification), the majority of them were underweight. Regarding the comparison between Ideal figure and Feel figure using the silhouette chart, the results showed that the participants preferred to have a skinny physique. Therefore, the mean FID values were found to be positive, thus reflecting higher dissatisfaction. This finding corroborates that reported in Zaccagni et al.'s (2014) study in which the participants preferred a skinny physique.

Studies have shown that body size dissatisfaction could lead to harmful conduct and poor mental health (Mintem et al., 2015; Zaccagni et al., 2014). Many factors have been reported as antecedents of body image dissatisfaction among individuals. Factors that are related to different groups, habits, traditions, and beliefs coupled with contemporary life anxiety and pressure, have all been implicated (Amenyah \& Michels, 2016; Mintem et al., 2015; Zaccagni et al., 2014; Tiggemann \& Pennington, 1990).

In the present study, the finding on the association of participants' body image assessment with their actual body weight status revealed high significant differences across all the traits. However, there was a significant main effect of higher BMI on body traits (Feel, Ideal, FID, and FAI). This implies that high BMI 


\section{Toriola and Onagbiye}

scores were related to greater body traits in this sample. Obesity has been regarded as a measure of success and wealth, most especially in the developing world (Alipour, Farhangi, Dehghan \& Alipour, 2015). However, recent technological advancements and multi-sociocultural influences have tended to favour slimness as an ideal body image (Alipour et al., 2015).

Results of the multiple linear regression analysis undertaken in this study to examine whether the traits (Feel and Ideal figures) significantly predicted participants' actual BMI revealed a trend in which higher Feel figure perceptions, yielded increases in the participants' BMI. However, the higher the Ideal figure perceptions, the lower the BMI. Yates, Edman and Aruguete (2004) had previously reported a high correlation between BMI and body image perceptions. Furthermore, BMI is a major contributing factor to body image (McCabe \& Ricciardelli, 2003). Hudson (2008) stated that the BMI might be a consistent predictor of body image assessment in both the short and long term, especially among women who are diverse in terms of the interaction of social and economic factors, thereby corroborating our findings. However, women were often implicated to have high body image disapproval with increased BMI (Hudson, 2008; Sriniva et al., 2017; Radwan et al., 2019). This could be attributed to a disordered eating style which increases BMI causing body dissatisfaction and leading to detrimental body mass control tactics (Hudson, 2008; Newman, Sontag \& Salvato, 2006).

Importantly, obesity and overweight among Saudi women have been reported to be on the increase in recent years with strong relationships observed with prolonged lifestyle-related illnesses (Alhussaini et al., 2018). The reasons highlighted were the rapid socio-cultural modifications emanating from the economic boost in the region, changes in diet and eating habits reflecting a westernised lifestyle, all of which increase the tendency for obesity and overweight. Understanding the link between the body image, weight discrepancy, and body mass index among this population is a necessity. This could be helpful to design a weight management strategy to improve the well-being and quality of life of Saudi women, especially those within a university setting.

\section{Study limitations}

The findings of the present study should be interpreted cautiously based on certain constraints. First, as this study was conducted among a group of university female students in Riyadh, the findings cannot be generalised to all female university students across the country. Second, BMI remains the preferred index used worldwide to evaluate the incidence of obesity, but the fact that it is only a surrogate of body fat limits its applicability. Also, the study did not take into consideration plausible genetic body weight predispositions. Notwithstanding these limitations, the present study provides information that could be useful to 
address the increasing trend of obesity and its psychosocial predispositions in this region.

\section{Conclusion}

The findings of this study showed significant relationships between actual body weight and body image discrepancy among university female students in Riyadh, Saudi Arabia. It was also found that both Feel and Ideal figure traits predicted BMI among the sample. A wellness programme and policy intervention that seek to create awareness and educate the women about their body image, as well as the need for regular physical activity participation and healthy eating, should be instituted and promoted for university female students. Further studies involving larger samples are needed to clarify the findings.

\section{Acknowledgements}

The researchers would like to thank all the participants in this study.

\section{References}

ALAhmari, T., Alomar, A. Z., ALBeeybe, J., Asiri, N., ALAjaji, R., ALMasoud, R. \& Al-Hazzaa, H. M. (2019). Associations of self-esteem with body mass index and body image among Saudi college-age females. Eating and Weight Disorders-Studies on Anorexia, Bulimia and Obesity, 24(6), 1199-1207. doi:10.1007/s40519-017-0471-0

Albeeybe, J., Alomer, A., Alahmari, T., Asiri, N., Alajaji, R., Almassoud, R. \& Al-Hazzaa, H. M. (2018). Body size misperception and overweight or obesity among Saudi college-aged females. Journal of obesity, 2018, 5246915. https://doi.org/10.1155/2018/5246915

Alhussaini, A. A., Alsuwedan, H. I., Alnefaie, H. F., Almubrek, R. A., Aldaweesh, S. A., Anitha, L. \& Qassem, H. (2018). Self-perception of body image among Saudi females at princess Nourah University, Riyadh, Kingdom of Saudi Arabia. Nutritional Status, Diet Intake Body Compos, 24(1), 117-123.http://www.nutriweb.org.my/publications/mjn0024_1/MJN\%2024(1)_117-

123_2018\%20 (Asma\%20AA,\%20Hessah\%20IA,\%20Hessa\%20FA\%20et\%20al.).php

Alipour, B., Farhangi, M. A., Dehghan, P. \& Alipour, M. (2015). Body image perception and its association with body mass index and nutrient intakes among female college students aged 18-35 years from Tabriz, Iran. Eating and Weight Disorders-Studies on Anorexia, Bulimia and Obesity, 20(4), 465-471. doi:10.1007/s40519-015-0184-1

Alwan, H., Viswanathan, B., Paccaud, F. \& Bovet, P. (2011). Is accurate perception of body image associated with appropriate weight-control behavior among adolescents of the Seychelles. Journal of obesity, 2011;2011:817242. doi:10.1155/2011/817242

Amenyah, S. D. \& Michels, N. (2016). Body size ideals, beliefs and dissatisfaction in Ghanaian adolescents: sociodemographic determinants and intercorrelations. Public Health, 139, 112-120. doi:10.1016/j.puhe.2016.05.012 


\section{Toriola and Onagbiye}

As-Sa'edi, E., Sheerah, S., Al-Ayoubi, R., Tajaddin, W. \& Habeeb, H. (2013). Body image dissatisfaction: Prevalence and relation to body mass index among female medical students in Taibah University 2011. Journal of Taibah University Medical Sciences, 8(2), 126-133. https://doi.org/10.1016/j.jtumed.2013.05.001

Bouzas, C., Bibiloni, M.D.M., \& Tur, J.A. (2019). Relationship between body image and body weight control in overweight $\geq 55$-year-old adults: a systematic review. International journal of Environmental Research and Public Health, 16(9), 1622. doi:10.3390/ijerph16091622

Cole, T. J., Flegal, K. M., Nicholls, D. \& Jackson, A. A. (2007). Body mass index cut offs to define thinness in children and adolescents: international survey. British Medical Journal, 335(7612), 194. doi:10.1136/bmj.39281.439178.80

General Assembly of the World Medical Association (2014). World Medical Association Declaration of Helsinki: ethical principles for medical research involving human subjects. The Journal of the American College of Dentists, 81(3), 14-18. PMID: 25951678

Higgins, E.T. (1987). Self-discrepancy: A theory relating self and affect. Psychological Review, 94(3), 319-340. https://doi.org/10.1037/0033-295X.94.3.319

Hudson, C.L.F. (2008). The relationship of body image, body mass index and self-esteem to eating attitudes in a normal sample. Master of Arts in Psychology Thesis. Manchester, UK: University of Canterbury. http://dx.doi.org/10.26021/5827

Khalaf, A., Westergren, A., Berggren, V., Ekblom, Ö. \& Al-Hazzaa, H. M. (2015). Perceived and ideal body image in young women in southwestern Saudi Arabia. Journal of Obesity. https://doi.org/10.1155/2015/697163.

Marfell-Jones, M.J., Stewart, A.D. \& De Ridder, J.H. (2012). International Standards for Anthropometric Assessment. Lower Hutt, New Zealand: International Society for the Advancement of Kinanthropometry (ISAK).

McCabe, M. P. \& Ricciardelli, L. A. (2003). Body image and strategies to lose weight and increase muscle among boys and girls. Health Psychology, 22(1), 39-57. PMID: 12558200

Mintem, G. C., Horta, B. L., Domingues, M. R. \& Gigante, D.P. (2015). Body size dissatisfaction among young adults from the 1982 Pelotas birth cohort. European Journal of Clinical Nutrition, 69(1), 55-61. doi:10.1038/ejen.2014.146

Newman, D. L., Sontag, L. M. \& Salvato, R. (2006). Psychosocial aspects of body mass and body image among rural American Indian adolescents. Journal of Youth and Adolescence, 35(2), 265275. https://doi.org/10.1007/s10964-005-9011-8

Radwan, H., Hasan, H. A., Ismat, H., Hakim, H., Khalid, H., Al-Fityani, L., ... \& Ayman, A. (2019). Body mass index perception, body image dissatisfaction and their relations with weight-related behaviors among university students. International Journal of Environmental Research and Public Health, 16(9), 1541. doi:10.3390/ijerph16091541

Srinivas, N., Ravi, M. R., Prashantha, B. \& Prakash, B. (2017). Prevalence of overweight and obesity, body image perception and weight control practices among college going adolescent girls in Mysore District, Karnataka. International Journal of Community Medicine and Public Health, 4(4), 954-958. DOI:http://dx.doi.org/10.18203/2394-6040.ijcmph20170950 
Strauman, T.J., Vookles, J., Berenstein, V., Chaiken, S. \& Higgins, E.T. (1991). Self-discrepancies and vulnerability to body dissatisfaction and disordered eating. Journal of Personality and Social Psychology, 61(6), 946-956. doi:10.1037//0022-3514.61.6.946

Tiggemann, M. \& Pennington, B. (1990). The development of gender differences in body-size dissatisfaction. Australian Psychologist, 25(3), 306-313. DOI:10.1080/00050069008260025

Yates, A., Edman, J. \& Aruguete, M. (2004). Ethnic differences in BMI and body/selfdissatisfaction among Whites, Asian subgroups, Pacific Islanders, and African-Americans. Journal of Adolescent Health, 34(4), 300-307. doi:10.1016/j.jadohealth.2003.07.014

Zaccagni, L., Masotti, S., Donati, R., Mazzoni, G. \& Gualdi-Russo, E. (2014). Body image and weight perceptions in relation to actual measurements by means of a new index and level of physical activity in Italian university students. Journal of Translational Medicine, 12(1), 42. doi:10.1186/1479-5876-12-42 